\title{
Angiostrongylus costaricensis, un parásito neotropical cuyo diagnóstico y tratamiento siguen siendo controversiales
}

\author{
Gustavo Gutiérrez-Espeleta1 ${ }^{1}$, Rolando Páez ${ }^{2} \&$ María Laura Arias Echandi ${ }^{3}$ \\ 1. Escuela de Biología, Universidad de Costa Rica; gustavo.gutierrez@ucr.ac.cr \\ 2. Hospital Calderón Guardia, Caja Costarricense de Seguro Social; rapaezsa@yahoo.com \\ 3. Centro de Investigación en Enfermedades Tropicales (CIET) y Facultad de Microbiología, Universidad de Costa Rica; \\ maria.ariasechandi@ucr.ac.cr
}

Recibido 15-XI-2017. Corregido 18-XII-2018. Aceptado 17-I-2019.

\begin{abstract}
Angiostrongylus costaricensis, a neotropical parasite with a controversial diagnosis and treatment. Abdominal angiostrogyliasis is a sporadic parasitosis that occurs in Neotropical countries, mainly in Costa Rica. It is a disease with an infrequent diagnosis and a controversial treatment. We discuss a case of an adult that presents a severe generalized discomfort, abdominal pain and an intense eosinophilia that leads to the detection of a mass at the right lower quadrant. Definitive diagnosis is achieved by the Morera Test and the treatment includes the use of azythromicin and metronidazole: no antihelmintic drugs or surgery were used. The case shows that both the diagnosis and treatment of this parasitosis are uncertain. An important underreporting of the disease may occur, as well as confusion of the symptoms with other pathologies.
\end{abstract}

Key words: Angiostrongylus costaricensis; diagnostis; treatment.

Gutiérrez-Espeleta, G., Páez, R. \& Arias Echandi, M. L. (2019). Angiostrongylus costaricensis, un parásito neotropical cuyo diagnóstico y tratamiento siguen siendo controversiales. Revista de Biología Tropical, 67(2) Suplemento, S159-S163.

La angiostrongilosis abdominal es una parasitosis esporádica, que ocurre en países latinoamericanos y especialmente en Costa Rica, cuyo primer caso fue observado en 1952 (Morera, 1987) y que para el año 2014, se habían descrito ya 198 casos (Romero-Alegría et al., 2014).

Es una parasitosis cuyo diagnóstico es infrecuente, no solo por tratarse de una enfermedad olvidada, de la cual hay gran desconocimiento así como ausencia de pruebas diagnósticas exactas y sensibles (Romero-Alegría et al., 2014).

Esta enfermedad es causada por un nemátodo del género Angiostrongylus, del cual se han descrito al menos 14 especies siendo dos patógenas para el ser humano: A. cantonensis asociado a meningoencefalitis eosinofílica
(Morera, 1970) y A. costaricensis, descrita por Morera y Céspedes (1971) en Costa Rica, responsable de síntomas abdominales que incluyen una intensa respuesta inflamatoria en la mucosa ileocecal, seguida por una reacción granulomatosa con una marcada eosinofilia y estenosis intestinal (Romero-Alegría et al., 2014). Todo lo anterior podría ser confundido con un cuadro clínico similar a una apendicitis aguda. La mayoría de casos se presentan como una enfermedad aguda, que requiere una laparotomía urgente (Rodríguez et al., 2008).

El ciclo de vida de este parásito es indirecto, e involucra a diversos moluscos que actúan como huéspedes intermediarios y roedores como huéspedes definitivos (Monge, Arroyo, \& Solano, 1978). El ser humano, e inclusive algunos otros primates, son hospederos 
accidentales y se infectan (Morera, 1970) al ingerir el molusco de manera completa o bien su moco que contiene larvas en estadio larval L3 (Arroyo \& Morera, 1978; Bonetti \& GraeffTexeira, 1998). Esta larva penetra los vasos linfáticos mesentéricos, migra al conducto torácico superior y finalmente se establece a nivel de arteriolas mesentéricas. Dado que el ser humano es un hospedero accidental, el parásito no puede completar su ciclo de vida y más bien los huevecillos se acumulan en las arteriolas asociadas al intestino (Kramer, 1998; Incani, Caleiras, Martín, \& González, 2007).

Este acúmulo de huevecillos provoca una intensa respuesta inmune, caracterizada por una eosinofilia marcada así como también una falta de perfusión a nivel intestinal, lo cual deriva en isquemia y necrosis tisular (Graeff-Teixera, Camilo-Coura, \& Lenzi, 1991a; Mesén \& Calvo, 2010).

El diagnóstico de este parásito se confirma mediante la identificación de huevecillos, larvas o formas adultas en especímenes quirúrgicos. No obstante, es frecuente la ausencia de elementos parasíticos, por lo que el diagnóstico puede basarse en la identificación histológica de infiltración masiva de la pared intestinal por eosinófilos, una reacción granulomatosa y una vasculitis eosinofílica que afecta arterias, venas, capilares y conductos linfáticos.

El diagnóstico indirecto y presuntivo puede ser llevado a cabo por pruebas serológicas que incluyen el test de Morera entre otros (Araya, Quesada, \& Vargas, 2015), análisis que se realiza en Costa Rica desde los años 1980. Es una prueba cualitativa, de aglutinación en látex y que utiliza antígeno crudo de formas adultas del parásito (Mesén \& Calvo, 2010; Araya et al., 2015). Esta prueba es barata y rápida y tiene una alta sensibilidad, no obstante su especificidad es cuestionable (Mesén \& Calvo, 2010).

$\mathrm{Su}$ tratamiento es controversial. Algunos autores reportan la resolución del cuadro con el uso de antihelmínticos, incluyendo tiabendazol, mebendazol o dietilcarbamizina a altas dosis (Araya et al., 2015). Sin embargo, otros autores no los recomiendan ya que incrementan el proceso inflamatorio al producirse la muerte y degeneración del parásito. La "Food and Drug Administration" (FDA) de los Estados Unidos, no avala el tratamiento con estos desparasitantes por no haberse logrado documentar con eficacia (Morera, Pérez, Mora, \& Castro, 1982). Otros autores se inclinan por la remoción quirúrgica del tejido infectado en casos agudos (Pizarro-Porras, 2003; Mesén \& Calvo, 2010) o únicamente por un tratamiento de soporte y observación de la evolución del paciente a través del tiempo siempre y cuando no se presente un cuadro abdominal agudo (Rodriguez, 1997; Graeff-Teixeira, Agostini, \& Rodríguez, 2007).

El presente estudio reporta un caso de angiostrongiliasis abdominal no aguda en un adulto costarricense que ilustra el vacío existente tanto con el diagnóstico como con el tratamiento de esta parasitosis.

\section{CASO}

Paciente masculino de 51 años de edad, profesional y originario del Valle Central de Costa Rica, sin antecedentes médicos o quirúrgicos y en buena condición física. A inicios del 2017, el paciente refiere dolor en el cuadrante inferior derecho abdominal, asociado a malestar, cansancio, anorexia, náuseas y fiebre acompañada de escalofríos. A la palpación el paciente presenta dolor y se detecta masa así como distensión abdominal. Después de unas tres semanas con estos síntomas, se registra una pérdida de peso de al menos ocho libras. Se realiza un hemograma el cual revela una marcada leucocitosis $(22000 / \mathrm{uL})$ acompañada de una evidente eosinofilia (52\%). El análisis seriado de heces, directo y por concentración no muestra ningún parásito y el coprocultivo resulta negativo. De la misma manera, se encuentra una alteración en sus enzimas hepáticas (ALT $78 \mathrm{U} / \mathrm{L}$, AST $36 \mathrm{U} / \mathrm{L}$, fosfatasa alcalina 285 U/L y GGT 75 U/L). Un primer ultrasonido abdominal realizado cuatro semanas después del inicio de los síntomas muestra un engrosamiento circunferencial de una porción del colon ceco-ascendente, que compromete una longitud de $8 \mathrm{~cm}$. También, un aumento de la 
ecogenicidad de la grasa pericolónica y mínima cantidad de líquido alrededor.

El análisis serológico o prueba de Morera se realizó también cuatro semanas después del inicio de los síntomas y fue positivo por A. costaricensis $(++++)$. Se decide iniciar el tratamiento con azitromicina $500 \mathrm{mg}$ y metronidazol (1500 mg) por diez días así como hacer un seguimiento del nivel de leucocitos y eosinófilos de manera semanal. De la misma manera se realiza una tomografía axial computarizada (TAC) la cual coincide con los cambios reportados en el ultrasonido exceptuando que circunscribe la lesión de colon a $5 \mathrm{~cm}$ de longitud con un espesor de $13 \mathrm{~mm}$.

Los valores hematológicos iniciaron una disminución paulatina llegando a normalizar luego de diez semanas. Las valoraciones serológicas también presentaron una disminución, no obstante la prueba de Morera continúa positiva $(+)$ al igual que la sintomatología de inflamación en el cuadrante inferior derecho.

\section{DISCUSIÓN}

La angiostrogilosis abdominal es una infección zoonótica emergente, de difícil diagnóstico y prevención (Incani et al., 2007) y poco estudiada por diversas causas, incluyendo el hecho de que muchas de sus infecciones son subclínicas y no requieren atención médica, existe un vacío importante en las metodologías utilizadas para su diagnóstico y existe poca literatura al respecto (Romero-Alegría et al. 2014). No obstante, debido a los cambios sociales dados en las últimas décadas, y a la globalización, el patrón epidemiológico de esta enfermedad ha cambiado (Romero-Alegría et al. 2014).

Se trata de una parasitosis que presenta un espectro clínico y patológico muy diverso, desde una forma aguda, donde se da daño ileocecal al punto donde se hace indispensable la cirugía, hasta casos donde se da su resolución a lo largo del tiempo sin llegar a tratamientos invasivos.

Cabe destacar, que la forma aguda es la que predomina en la práctica. Las lesiones intestinales dadas por la presencia del parásito en las arterias meséntéricas resultan en isquemia intestinal, especialmente en el segmento ileocecal (Rodríguez et al., 2008). Esto conduce a un espectro de eventos finales que puede variar entre infarto intestinal, perforación intestinal, formación de un pseudotumor y apendicitis aguda (Graeff Teixera, Camilo-Coura, \& Lenzi, 1991b; Graeff-Teixeira et al., 2007).

Debido al comportamiento errático de este parásito, su localización anatómica en el huésped, incertidumbre clínica y el poco número de referencias bibliográficas asociadas (Wu, French, \& Turner, 1997; Voung et al., 2002), su manejo y diagnóstico es complicado. Actualmente, el diagnóstico definitivo depende del análisis histológico de biopsias intestinales (Mesén \& Calvo, 2010). Existen muy pocas pruebas serológicas validadas, con sensibilidades y exactitudes variables. La aparición de falsos negativos aún en casos agudos es frecuente especialmente en pacientes infectados con bajos niveles de anticuerpos.

En Costa Rica el diagnóstico serológico se realiza, desde los años ochenta, por medio de la Prueba de Morera, una prueba cualitativa. También en los últimos años se han estandarizado dos pruebas adicionales que incluyen inmunofluorescencia indirecta para detectar anticuerpos IgG e IgG1 (Mesén \& Calvo, 2010)

En cuanto al tratamiento de esta parasitosis, y en aras de resolver la isquemia provocada por el parásito, la cirugía es indicada en pacientes que presenten un cuadro de abdomen agudo. Por otro lado, el uso de medicamentos para esta parasitosis es muy diverso y poco uniforme, variando desde aquellos médicos que recomiendan el uso de antihelmínticos hasta los que únicamente hacen tratamiento de soporte, evadiendo el uso de antiparasitarios y esperando la disminución de síntomas (Graeff-Teixeira et al., 2007) y eventual autolimitación. No obstante, el criterio de cura no ha sido fácil de establecer (Loria-Cortés \& Lobo-Sanahuja, 1980).

Diversos antiparasitarios incluyendo metronidazol, ivermectina, mebendazol, albendazol, tiabendazol y albendazol, entre otros, (Morera \& Bontempo, 1985) han sido evaluados como potenciales agentes terapéuticos 
para esta infección, no obstante los efectos han sido variables y hasta el momento no ha sido comprobada la eficacia de su uso. Por otro lado, existe el temor de que estos productos se asocien a migración errática del parásito (Morera \& Bontempo, 1985) o empeoramiento de las lesiones existentes (Terada, Kino, Akyol, \& Sano, 1993).

El caso descrito anteriormente pone de manifiesto que el diagnóstico y el tratamiento de esta enfermedad parasitaria continúan siendo inciertos. No se descarta que exista un subregistro de esta parasitosis o que los síntomas hayan sido confundidos con otra patología. Por esta razón, es de gran importancia concientizar al cuerpo médico de la existencia de este parásito y familiarizarlo de su sintomatología específica, así como de fomentar el desarrollo de metodologías diagnósticas certeras. En cuanto al tratamiento, es muy importante evaluar y estandarizar el uso de antihelmínticos en esta parasitosis, y llegar a determinar si su uso es adecuado o no en el tratamiento de pacientes que no requieran de la cirugía.

Declaración de ética: los autores declaran que todos están de acuerdo con esta publicación y que han hecho aportes que justifican su autoría; que no hay conflicto de interés de ningún tipo; y que han cumplido con todos los requisitos y procedimientos éticos y legales pertinentes. Todas las fuentes de financiamiento se detallan plena y claramente en la sección de agradecimientos. El respectivo documento legal firmado se encuentra en los archivos de la revista.

\section{RESUMEN}

La angiostrongilosis abdominal es una parasitosis esporádica, que ocurre en países del neotrópico, especialmente en Costa Rica. Es una enfermedad cuyo diagnóstico es infrecuente y su tratamiento controversial. Se presenta un caso de un adulto que muestra un malestar generalizado, dolor abdominal y una intensa eosinofilia que lleva a la detección de una masa en la fosa iliaca inferior derecha. El diagnóstico definitivo se hace mediante la prueba de Morera y el tratamiento incluye el uso de azitromicina y metronidazol, no obstante no se usan antihelmínticos ni se realiza cirugía. El caso descrito pone de manifiesto que tanto el diagnóstico como el tratamiento de esta enfermedad parasitaria continúan siendo inciertos. No se descarta que exista un subregistro importante de esta parasitosis o que los síntomas sean confundidos con otras patologías.

Palabras clave: Angiostrongylus costaricensis; diagnóstico; tratamiento.

\section{REFERENCIAS}

Araya, A., Quesada, L. \& Vargas, H. (2015). Angiostrongilosis abdominal. Revista Médica Costa Rica y Centro América. LXXI, 711-718.

Arroyo, R., \& Morera, P. (1978). Viability of the first stage larvae of Angiostrongylus costaricensis in rat feces. Journal of Parasitology, 64(1), 146.

Bonetti, V. C., \& Graeff-Teixeira, C. (1998). Angiostrongylus costaricensis and the intermediate hosts: observations on elimination of L3 in the mucus and inoculation of L1 through the tegument of mollusc. Revista Sociedade Brasileira de Medicina Tropical, 31, 289-294.

Graeff-Teixeira, C., Agostini, A., \& Rodríguez, R. (2007). Angiostrongiliase abdominal. In: W. Tavares \& L. A. C. Marinho (Eds.), Rotinas de diagnótico e tratamento das doencas infecciosas e parasitárias (pp. 98-101) Sao Paulo: Atheneu.

Graeff-Teixeira, C., Camilo-Coura, L., \& Lenzi, H. L. (1991a). Abdominal angiostrongyliasis: an underdiagnosed disease. Memorias Instituto Oswaldo Cruz, 82, 353-354.

Graeff-Teixeira, C., Camillo-Coura, L., \& Lenzi, H. L. (1991b). Clinical and epidemiological aspects of abdominal angiostrongyliasis in southern Brazil. Revista Instituto Medicina Tropical de Sao Paulo, 33, 373-378.

Incani, R. N., Caleiras, E., Martín, M., \& González, C. (2007). Human infection by Angiostrongylus costaricensis in Venezuela: first report of a confirmed case. Revista Instituto Medicina Tropical de Sao Paulo, 49, 197-200.

Kramer, M. H. (1998). First reported outbreak of abdominal angiostrongyliasis. Clinical Infectious Diseases, 26, 365-372.

Loría-Cortés, R., \& Lobo-Sanahuja, J. (1980). Clinical abdominal angiostrongyliasis. A study of 116 children with intestinal eosinophilic granuloma caused by Angiostrongylus costaricensis. American Journal of Tropical Medicine and Hygiene, 29, 538-544.

Mesén, P., \& Calvo, N. (2010). Diagnóstico de la angiostrongilosis abdominal en Costa Rica. Costa Rica: CNRP-INCIENSA. 
Monge, E., Arroyo, R., \& Solano, E. (1978). A new definitive natural host of Angiostrongylus costaricensis. Journal of Parasitology, 64(1), 34.

Morera, P. (1970). Investigación del huésped definitivo de Angiostrongylus costaricensis Morera y Céspedes 1971. Boletín Chileno de Parasitología, 25, 133-134.

Morera, P. (1987). Abdominal angiostrongyliasis. Baillere's Clinical Tropical Medicine and Communicable Diseases, 2, 747-753.

Morera, P. \& Bontempo, I. (1985). Acción de algunos antihelmínticos sobre Angiostrongylus costaricensis. Revista Médica Hospital Nacional de Niños, 20, 165-174.

Morera, P., \& Céspedes, R. (1971). Angiostrongylus costaricensis $\mathrm{n}$. sp (Nematoda: Metastrongyloidea), a new lungworm occurring in man in Costa Rica. Revista Biología Tropical, 18(1-2), 173-185.

Morera, P., Pérez, F., Mora, F., \& Castro, L. (1982). Visceral larva migrans-like síndrome caused by Angiostrongylus costaricensis. American Journal of Tropical Medicine and Hygiene, 31, 67-70.

Pizarro-Porras, J. (2003). Angiostrongiliasis abdominal. Revisión de 33 casos. (Tesis de Licenciatura). Universidad de Costa Rica, Sede Rodrigo Facio.
Rodríguez, R. (1997). Anatomía patológica de angiostrongilose abdominal (Disertación de Maestado). Universidade Federal Fluminense, Brasil.

Rodríguez, R., Martins, R., Peruzzo, L., Moacir, P., Garcia, E., \& Fornari, F. (2008). Abdominal angiostrongyliasis: report of two cases with different clinical presentations. Revista Instituto Medicina Tropical de Sao Paulo, 50, 339-341.

Romero-Alegría, A., Belhassen-García, M., Velasco-Tirado, V., García-Mingo, A., Alvela-Suárez, L., PardoLledias, J., \& Cordero, M. (2014). Angiostrongylus costaricensis: Systemtic review of case reports. Advances in Infectious Disease, 4, 36-41.

Terada, M., Kino, H., Akyol, C., \& Sano, M. (1993). Effects of mebendazole on Angiostrongylus costaricensis in mice with special reference to the timing of treatment. Parasitic Research, 79, 441-443.

Vuong, P., Brama, P., Bonete, R., Houissa-Vuong, S., Catanzano-Laroudie, M., \& Baviera, E. (2002). Necrotic eosinophilic angiitis with ileal perforation and peritonitis secondary to abdominal angiostrongyliasis. Presse Médicale, 31, 1700-1703.

Wu, S., French, S. \& Turner, J. (1997). Eosinophilic ileitis with perforation caused by Angiostrongylus costaricensis. A case study and review. Archives of Pathology and Laboratory Medicine, 121, 989-991. 